\title{
PERFIL EPIDEMIOLÓGICO DOS PACIENTES ATENDIDOS NO SETOR DE FISIOTERAPIA EM ORTOPEDIA E TRAUMATOLOGIA DA CLÍNICA ESCOLA DE FISIOTERAPIA DA UNIVERSIDADE SÃO FRANCISCO \\ EPIDEMIOLOGICAL PROFILE OF PATIENTS ATTENDED IN THE ORTHOPEDICS AND \\ TRAUMATOLOGY PHYSICAL THERAPY SECTOR AT UNIVERSIDADE SÃO FRANCISCO
}

\author{
DAVID, Gustavo Pelaes ${ }^{1}$ COSTA, Patrícia Teixeira ${ }^{1}$, FRAGA SOUZA, Grazielle Aurelina ${ }^{1}$, \\ FUSARO, Claudio ${ }^{1}$ \\ ${ }^{1}$ Universidade São Francisco \\ claudio.fusaro@usf.edu.br
}

\begin{abstract}
RESUMO. O objetivo do estudo foi avaliar o perfil dos pacientes que apresentam disfunções ortopédicas, traumatológicas e reumatológicas atendidos no período compreendido entre fevereiro de 2015 a dezembro de 2016, na clínica-escola do curso de fisioterapia da Universidade São Francisco. Trata-se de um estudo de caráter retrospectivo, com informações colhidas nos prontuários do setor de Fisioterapia em Ortopedia e Traumatologia da Clínica Escola de Fisioterapia da Universidade São Francisco no período compreendido entre fevereiro de 2015 e dezembro de 2016. Verificou-se que a idade média de acometimento foi de $48 \pm 35$ anos, com maior prevalência do sexo feminino, com $57,47 \%$. A região mais acometida foi a região do joelho, com $20 \%$ dos casos, e a origem de encaminhamentos foi o HUSF, com 39,08\%. Conclui-se que o perfil do paciente atendido na Clínica-Escola de Fisioterapia da Universidade São Francisco é do gênero feminino com acometimento do membro inferior, sendo prioritariamente encaminhado pelo serviço do HUSF.
\end{abstract}

Palavras - Chave: Fisioterapia, Perfil epidemiológico, Análise retrospectiva

ABSTRACT. The aim of the study was to evaluate the patients' profiles with orthopedic, traumatological and Rheumatologic disorders attended between February 2015 and December 2016 at the Clinical of Physiotherapy course of the University São Francisco. A retrospective study was carried out with the medical records collected in the Physiotherapy sector in Orthopedics and Traumatology of the Clinical School of Physiotherapy of the University São Francisco in the period between February 2015 and December 2016. We can verify that the average age of involvement was $48 \pm 35$ years old, with a higher prevalence of females, with a proportion of $57.47 \%$. The most affected region was the knee, being $20 \%$, and the most incident origin of referrals was HUSF, with $39.08 \%$. It is concluded that the profile of the patient attended at the Clinic-School of Physical Therapy of the University São Francisco is the female gender with involvement of the lower limb, being primarily referred by the HUSF service..

Keywords: Physical Therapy, Epidemiologic Profile, Retrospective analysis

\section{INTRODUÇÃO}

Os avanços na área da saúde nos últimos tempos provocaram um impacto positivo na expectativa de vida da população mundial, isso tem sido observado de forma geral na população brasileira em função dos avanços na área médica, da produção de vacinas, do desenvolvimento de novos medicamentos, dos atendimentos complementares que proporcionam uma melhoria 
na qualidade de vida, porém, ainda assim, a partir da década de oitenta causas externas passaram a constituir um grave problema de saúde pública (LEMOS et al.,2013).

As disfunções musculoesqueléticas são compreendidas como as doenças do sistema locomotor e também do tecido conectivo. Estas doenças são a causa mais comum de incapacidade crônica ao redor do mundo. Diversas doenças do gênero são predominantemente genéticas, mas também podemos verificar que outras surgem pela associação de fatores genéticos àqueles ambientais, podemos citar associadas à idade e outros que se estabelecem sob à influência de fatores biológicos e/ou psicossociais, estes últimos, por sinal, ultimamente muito citados e influentes em estudos dentro da área da fisioterapia em ortopedia e traumatologia (CONNELLY; WOOLF; BROOKS, 2006; GRAFFITHS, 2002).

A impactação econômica destas condições é muito grande, não somente pelos gastos diretos resultantes dos tratamentos demandados, bem como pela consequente perda parcial, por vezes, significativa da produtividade do sujeito que está acometido. Estes fatores levam ao comprometimento real de diversos aspectos: aspectos sociais e emocionais (como citado anteriormente, os aspectos psicossociais, muito relatados atualmente na abordagem terapêutica cognitivo-comportamental (WOOLF; ERWIN; MARCH, 2012).

O principal objetivo da pesquisa epidemiológica é o de proporcionar compreensão dos fenômenos que se relacionam à saúde das diversas populações, e deve nos servir de norteamento inicial para as estratégias de desenvolvimento de ações que se interessem por modificar os diversos padrões relacionados ao desencadeamento de doenças (BICALHO; BARROS-FILHO, 2003).

Os estudos de levantamentos epidemiológicos têm importância crucial e corroboram para o estabelecimento das demandas dos serviços de uma determinada comunidade. Na sua essência, deve produzir apontamentos e reflexões profundas, não somente ao poder público, mas também aos coordenadores e dirigentes de serviços que prestam atendimento às comunidades, incluindo-se aqui o foco deste trabalho que é o atendimento da fisioterapia em ortopedia, traumatologia e reumatologia, e espera-se que da reflexão dos dirigentes resultem planejamento e organização de políticas públicas em saúde, tanto quanto, remanejamento e organização dos serviços prestados a comunidade afim de contemplar as reais demandas que são insurgentes no cotidiano das populações onde tais serviços são relevantes (UENO HM, NATAL D., 2008; GOTLIEB SLD, LAURENTI R, JORGE MHPM, 2012).

$\mathrm{O}$ atendimento fisioterapêutico ambulatorial ofertado pelo SUS ainda é muito pequeno e com desigualdades regionais. A remuneração é inadequada e em grande parte, ocorrem por meio de convênios com estabelecimentos privados. Um estudo brasileiro de base populacional identificou que a prevalência de utilização dos serviços de fisioterapia ao longo da vida foi de $30,2 \%$, isso mostra que há uma necessidade de pesquisas para fornecer à literatura quais as frequências e tipos de utilização dos serviços prestados (SILVA; SIRENA,2012).

A reabilitação em saúde pública apresenta uma conjuntura atual, em sua maioria, de desarticulação e desigualdade, não conseguindo atender as demandas das populações em procura dos serviços, ou seja, é uma demanda deficitária ao acesso à fisioterapia, e muitas vezes, a não obtenção de resolutividade dos problemas, entenda-se aqui problemas como as queixas e disfunções apresentadas pelos pacientes quando procuram o atendimento primário e de aplicação generalista, o que faz ampliar veementemente o fluxo de pacientes para o serviço especializado de fisioterapia em ortopedia, traumatologia e reumatologia. Tal fato, em associação ao fluxo de atendimento em centros de especialidades médicas, também ao fluxo de atendimento pós-operatório de serviços hospitalares, convergem tais fatores para a altíssima demanda de pacientes com disfunções outrora denominadas como disfunções músculoesqueléticas, aqui agora, discriminando-as como disfunções de origem ortopédica, traumatológica e reumatológicas para os serviços de fisioterapia. Esta demanda é extremamente alta e ficam claras as dificuldades em atender a tal fluxo de pacientes quando observamos em nosso levantamento de dados o alto número de pacientes em fila de espera, aguardando vagas 
para o atendimento especializado nesta área que aborda o sistema músculo-esquelético (RIBEIRO et. al., 2010; CARDOSO, 2004).

Como em qualquer outro sistema biológico, o sistema músculo-esquelético não é estático, encontrando-se em constante equilíbrio, denomina-se esta condição de homeostase. Quando o sistema é submetido a um estresse externo ou uma força, responderá de maneira bastante específica objetivando restabelecer o estado de equilíbrio. As doenças e lesões do sistema músculo-esquelético causam dor, podem levar a deformidades e até mesmo à perda de funções, causando a incapacitação de um maior número de pessoas do que aquelas afetadas em outro sistema. Há uma estimativa de que $40 \%$ dos indivíduos manifestarão dor músculoesquelética crônica, esteja envolvida em traumas ou não (WATANABE,2012).

Tal homeostase citada há pouco pode ser abruptamente violada quando o sistema músculo-esquelético é exposto as mais diversas condições relacionadas à vida urbana. $\mathrm{O}$ aumento do número de veículos nas grandes cidades resultou em maior número de acometimentos ortopédicos e traumáticos e internações hospitalares, tanto no serviço de prontosocorro quanto no atendimento continuado. Em função disso o trauma chegou ao primeiro lugar das etiologias de morbimortalidade em pessoas entre os 0 a 39 anos de idade, sendo responsável por 150.000 mortes por ano, podendo esse número ser triplicado se considerado o número de vítimas inválidas permanentemente. Nesse cenário as lesões do sistema músculo-esquelético ocorrem em até $85 \%$ dos pacientes que são vítimas de trauma fechado, isto é, traumas que não ocorrem exposição, não causando risco à vida, mas que necessitam de intervenções para que não se coloque a parte afetada em perigo (WATANABE, 2012).

As lesões ortopédicas traumáticas podem ser divididas em quatro tipos: contusão (lesão traumática do tegumento); entorse (lesão traumática das partes moles); fratura (lesão óssea traumática) e lesão articular traumática (existe perda parcial ou total do contato entre os ossos) (OLIVEIRA; BRAGA, 2010).

Ainda que na ortopedia existam as doenças degenerativas. Tais lesões podem levar a perda de função, causar dores, inflamações e limitações das atividades de vida diárias. Essas doenças ocorrem nas estruturas que fazem a ligação entre os ossos, podendo ser formadas por diversos tecidos. As patologias mais comumente vistas na clínica: Osteoatrose; Artrite Reumatóide do Adulto; Osteoporose; Osteopenia (COELHO, 2005).

Dentro das lesões ortopédicas podem-se citar as lesões de tecidos moles, tais lesões ocorrem de maneira mais comum na pele, tendões, ligamentos e bolsas sinoviais. Essas lesões são classificadas em: Contusão; Distensão; Tendinite; Entorse; Luxação e Subluxação; Lacerações, Sinovites e Síndromes Miofasciais (SILVA, 2015).

De acordo com pesquisa realizada na Universidade do Sul de Santa Catarina citaram como lesões mais frequentes, a Síndrome do impacto do ombro com atendimento de 54 casos, representando $74,3 \%$ do total de casos,, seguida de lombalgia/lombocitalgia com 54 casos, $73 \%$ do total , a pesquisa mostrou ainda que a artrose apareceu em 47 pessoas $(8,47 \%)$ seguidas das Fraturas com 40 casos, 7,21\% do total; Cervicalgia/ cervicobraquialgia com 35 casos, 6,31\% do total; Escoliose com 24 casos, 4,32\% do total; Entorses com 19 casos, 3,42\% do total; Epicondilite com 11 casos, 1,98\% do total; Fibromialgia com 10 casos, 1,8\% do total e Condromalácia patelar com 9 casos, 1,62\% do total (MARGOTTI , 2013).

Estudos na área de ortopedia vêm tendo destaque nos últimos anos por atuar na prevenção, no diagnóstico e no tratamento dos distúrbios do sistema músculo- esquelético que causam dores, deformidades e perda de função (OLIVEIRA; BRAGA, 2010).

De acordo com a UNIVERSIDADE SÃO FRACISCO (2013), a Fisioterapia como a maioria das profissões atuantes da área da saúde, teve sua relevância histórica em meados do século passado, no período pós-guerra. Foi a partir deste momento que se identificou a atuação deste profissional em sua concepção mais simples e primitiva, representada pela capacidade de restaurar funções perdidas, reinserindo o ser humano na sua realidade social e profissional. 
Não se têm dúvidas de que ao longo dos últimos 50 anos a dimensão da atuação do fisioterapeuta se ampliou muito. Considerando o panorama em que a fisioterapia vem escrevendo sua história, é notório o quanto as últimas décadas foram expressivas em termos de expansão de mercado de trabalho destes profissionais e do desenvolvimento de tecnologia e conhecimentos envolvidos nos recursos utilizados para avaliação, prevenção e promoção da saúde e reabilitação dos pacientes (UNIVERSIDADE SÃO FRACISCO, 2013).

A atuação do fisioterapeuta deixou de se limitar a hospitais e clínicas de reabilitação e se estendeu a novas áreas como indústrias, por meio de trabalhos de prevenção e ergonomia voltados à saúde do trabalhador; clubes, representado pelo papel preventivo e de reabilitação precoce de atletas e clínicas dermatológicas e de cirurgia plástica. Esta atuação também se especializou e se desenvolveu nas áreas de atenção primária, secundária e terciária com especialidades como ortopedia, traumatologia, reumatologia, neurologia, cardiologia, pneumologia, pediatria, geriatria, ginecologia, obstetrícia, urologia, enfermarias hospitalares e unidades de terapia intensiva (UNIVERSIDADE SÃO FRACISCO, 2013).

A Clínica de Fisioterapia do Campus Bragança Paulista oferece atendimento gratuito nas áreas de Ortopedia, Traumatologia e Reumatologia; Neurologia Adulto; Saúde da Criança e Adolescente; Cardiologia e Pneumologia; Saúde da Mulher e Urologia; Dermatofuncional e Desportiva

Com mais de 14 anos de atividade, reconhecida estrutura física e equipamento de última geração, a Clínica é referência na prestação de serviços à comunidade, bem como, por estar estabelecida no âmbito acadêmico com atividades de ensino, pesquisa e extensão. Desde sua fundação, já foram realizados mais de 9 mil atendimentos por meio de estágios curriculares, projetos de extensão e outras atividades acadêmicas (INSTITUCIONAL,2017).

Por meio destas disciplinas, o aluno tem a oportunidade de estagiar em áreas e especialidades distintas e obrigatórias de maneira a vivenciar a intervenção fisioterapêutica nos diferentes níveis de atenção à saúde e em locais que contemplam a prática terapêutica em diferentes níveis de complexidade (UNIVERSIDADE SÃO FRACISCO, 2013).

O cumprimento do Estágio Supervisionado passa por duas instâncias: a necessidade de estrutura física (instituições e serviços que possam abrigar os estágios) e a necessidade de recursos humanos (docentes supervisores de estágio), garantindo as orientações e supervisões constantes durante a realização dos estágios (UNIVERSIDADE SÃO FRACISCO, 2013).

Os espaços físicos necessários para que se concretize o aprendizado prático estão assegurados pela designação de área específica para a estruturação da Clínica Escola de Fisioterapia da Universidade São Francisco e pelas parcerias e convênios celebrados pelo curso para esta prática (UNIVERSIDADE SÃO FRACISCO, 2013).

Os recursos humanos necessários estão assegurados pela atribuição de horas a docentes/supervisores que são designados especificamente para orientar e supervisionar os alunos/estagiários nas diferentes áreas. Para a realização desta atribuição, existe a preocupação de garantir que ao menos um supervisor de cada área de estágio seja também o docente na disciplina de fisioterapia aplicada à especialidade correspondente de maneira a assegurar a interrelação entre a teoria e a prática, otimizando o processo de ensino-aprendizagem durante os estágios práticos supervisionados (UNIVERSIDADE SÃO FRACISCO, 2013).

As atividades desenvolvidas no estágio supervisionado compreendem desde a realização de reuniões clínicas para discussão de casos, seminários, redação e discussão de relatórios até o atendimento fisioterapêutico ao paciente propriamente dito. A correção e discussão dos relatórios de atividades são realizadas em horários compreendidos pela carga horária semanal do estágio, representando parte da avaliação do aluno conforme previsto no regulamento. A relação entre alunos e docentes para a supervisão deve ser de no máximo seis alunos por professor. As áreas de estágio propostas pelo Curso de Fisioterapia proporcionam ao aluno experiências diversificadas por meio de atuação em diferentes níveis de atenção e complexidade na área da saúde. Desta forma, a preocupação com a promoção da saúde bem 
como com a prevenção de doenças e suas sequelas é uma constante em todos os campos de estágio, independentemente do nível de complexidade dos mesmos (UNIVERSIDADE SÃO FRACISCO, 2013).

O presente trabalho objetiva determinar a incidência das diversas disfunções ortopédicas, traumatológicas e reumatológicas na Clínica Escola de Fisioterapia da Universidade São Francisco no período de fevereiro à dezembro de 2016, levantando as modalidades terapêuticas empregadas no tratamento dos pacientes usuários do serviço de fisioterapia da clínica escola para que os resultados do estudo possam contribuir para o entendimento do perfil dos pacientes atendidos e as modalidades mais utilizadas no tratamento dos pacientes.

\section{METODOLOGIA}

Trata-se de um estudo de caráter retrospectivo, realizado na Clínica-Escola de Fisioterapia da Universidade São Francisco no setor de Ortopedia e Traumatologia no período de Fevereiro de 2015 a Dezembro 2016.

O projeto foi aprovado pelo Comitê de Ética e Pesquisa da Universidade São Francisco (Parecer $\left.\mathrm{n}^{\circ} 1.798 .746\right)$

Para a realização da pesquisa todos os prontuários dos pacientes do setor mencionado acima foram utilizados, coletando-se destes as seguintes informações: idade, sexo, cidade de origem, diagnóstico clínico, origem do encaminhamento, número de sessões, tipo de tratamento (critérios de inclusão), informações que foram registradas em uma ficha de informações. Tais informações foram colhidas dos pacientes que estão na fila de espera do setor de Ortopedia e Traumatologia.

Os critérios de exclusão foram todos os prontuários que não continham alguma das informações acima

Os dados obtidos foram organizados em uma planilha do software Microsoft Excel (2016). Foi realizado os cálculos de média, desvio padrão, frequência e porcentagem.

\section{RESULTADOS E DISCUSSÃO}

Um total de 199 prontuários foram analisados, dos quais foram utilizados 174 prontuários, sendo 95 de pacientes que já haviam sido tratados ou estavam em tratamento na Clínica Escola e 79 de pacientes que se encontravam na lista de espera no setor de Fisioterapia em ortopedia e traumatologia. Foram excluídos 25 prontuários que não continham alguns dos requisitos estipulados como critérios de inclusão.

Os pacientes atendidos na Clínica-Escola de Fisioterapia da Universidade São Francisco no setor de Ortopedia e Traumatologia tem no gênero feminino a maior incidência de atendimento, com 100 pacientes $(57,47 \%)$, contra 74 (42,53\%) pacientes do gênero masculino. A média de idade é de 48,8 anos, apresentando desvio padrão de 18,2 2 , com faixa dos 13 anos até 86 anos.

A maior incidência que observar-se de pacientes do gênero feminino vem contrariar o que SILVA et.al. (2015) descreveram em seu estudo, apontando um resultado diferente após analisarem 274 prontuários, encontrando 147 pacientes do sexo masculino 55,5\% e 118 do sexo feminino 44,5\%. Essa observação de incidência do sexo masculino ser maior em número de atendimentos também foi demonstrada por CHAMLIAN et al (2013), quando apresentam em seu estudo que dos 474 prontuários analisados dos pacientes atendidos no Lar escola São Francisco, entre os anos de 2006 a 2012, 339 pacientes eram do sexo masculino. Esses dados comparativos entre estes dois estudos e o presente, é possível concluir que a procura pelo atendimento fisioterapêutico em relação ao gênero varia de acordo com o local e serviço, 
dependendo de características regionais e outros fatores epidemiológicos a serem investigados em outros estudos que possam vir a ser realizados.

Pode-se ainda correlacionar a média de idade com o estudo de FUNCK; ESTIVALET (2015), que realizaram um estudo referente ao perfil dos pacientes atendidos na fisioterapia da prefeitura do município de Boa Vista do Cadeado-RS, tendo uma maior prevalência nos indivíduos de 41 a 50 anos (20\%).

Através da Figura 1 pode-se observar que a maioria dos pacientes atendidos na ClínicaEscola de Fisioterapia da Universidade São Francisco reside em Bragança Paulista (n=149), seguido de Atibaia ( $\mathrm{n}=11)$, Outros que correspondem a cidades como Bom Jesus dos Perdões, Nazaré, Tuiuti e Socorro (n=6), Piracaia (n=3), Pinhalzinho (n=3), Joanópolis ( $n=2)$.

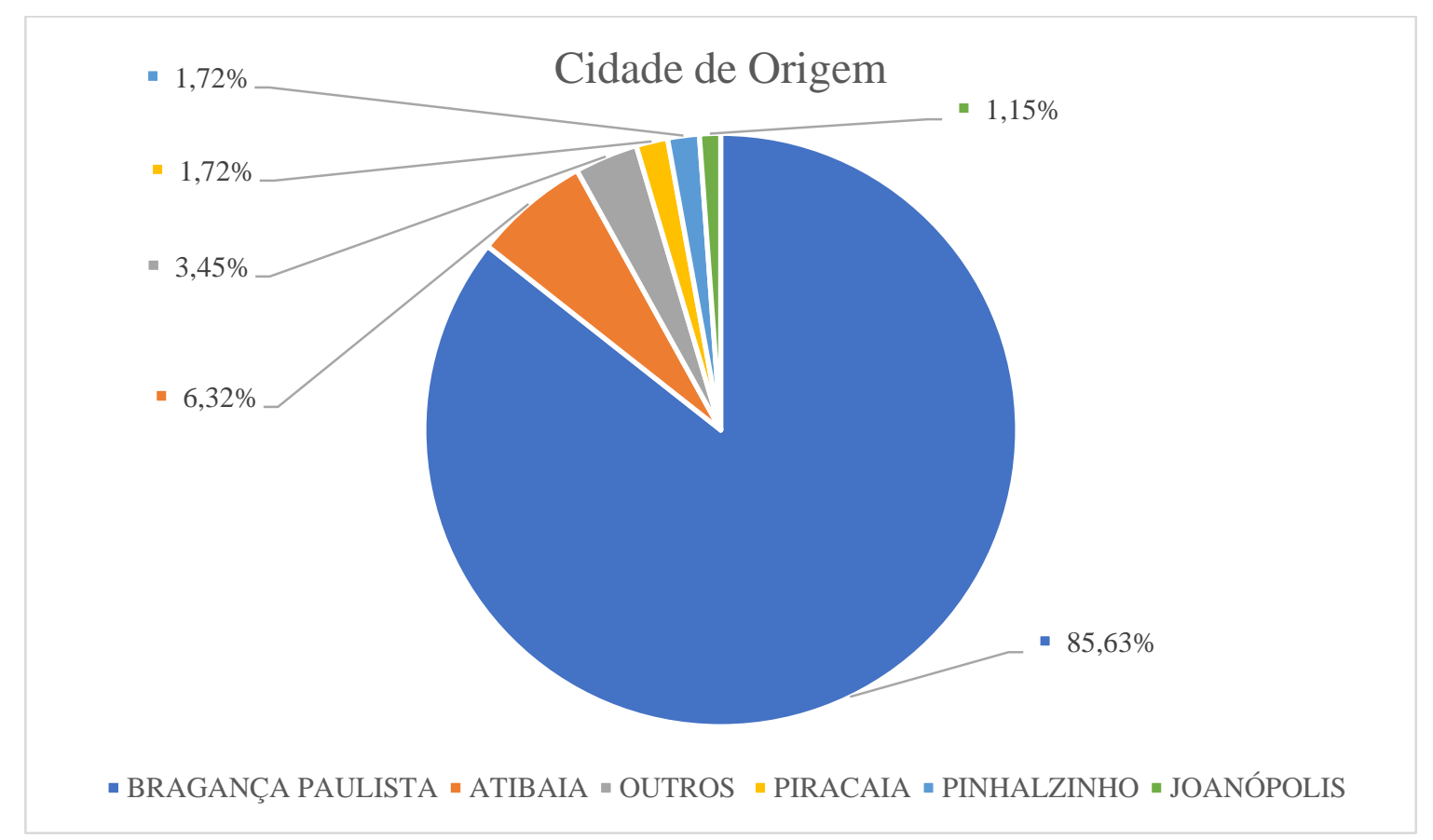

Figura 1 - Cidade de Origem.

Como o presente estudo demonstrou, o serviço prestado pela Clínica Escola de Fisioterapia da Universidade São Francisco atua de forma significativa na comunidade em que está inserida, promovendo um grande número de atendimentos aos moradores de Bragança Paulista e região, dessa forma, desempenhando importante papel social e no desenvolvimento de saúde da cidade. SILVA et. al. (2015), também relatam maior incidência dos pacientes residentes de Viçosa, local em que se situa a clínica de fisioterapia da pesquisa em questão. RAMOS et. al. (2012), relata um predomínio de pacientes residentes de Jequié-BA $(85,1 \%)$ que tratam na Clínica Escola de Fisioterapia da UESB. Podemos também correlacionar esses resultados com um maior número de encaminhamentos provenientes do HUSF, comprovando o atendimento local pelo predomínio de pacientes residentes de Bragança Paulista.

Em relação ao local de encaminhamento dos pacientes verificamos que o Hospital Universitário São Francisco (HUSF) foi o local com o maior número de encaminhamentos (=68), seguido do serviço municipal de Bragança Paulista $(n=50)$, Centro de Especialidade de Bragança Paulista $(n=21)$, Santa Casa de Misericórdia de Bragança Paulista $(n=8)$, convênios particulares $(n=2)$ e outros locais como hospitais de São Paulo, Itajubá, Jundiaí, clínicas particulares $(n=24)$ como demonstrado na Figura 2. Com relação as regiões acometidas pelas diversas patologias e disfunções, pode-se observar na Figura 3 que a região do joelho $(n=39)$, foi a qual mais incidiu acometimentos, seguido pela região do ombro $(n=28)$, coluna lombar $(n=24)$, acometimentos generalizados: $(A G)(n=18)$ que correspondem a patologias que acometem mais de um segmento corpóreo, a exemplo fibromialgia, osteoporose, artrite 
reumatoide juvenil, entre outros, seguido de tornozelo $(n=15)$, coluna cervical $(n=14)$, mão $(n=13)$, quadril $(n=10)$, braço $(n=8)$, coluna torácica $(n=7)$, antebraço $(n=6)$, perna $(n=6)$, pé $(n=5)$ e coxa $(n=2)$. Durante a coleta pode-se observar que vários pacientes apresentavam mais de uma patologia, totalizando 195 patologias de 174 prontuários, mas a maioria 152 pacientes $(87,35 \%)$, apresentam apenas uma patologia, 19 (10,91\%) pacientes apresentam 2 patologias, e 3 pacientes $(1,74 \%)$ apresentam 3 patologias.

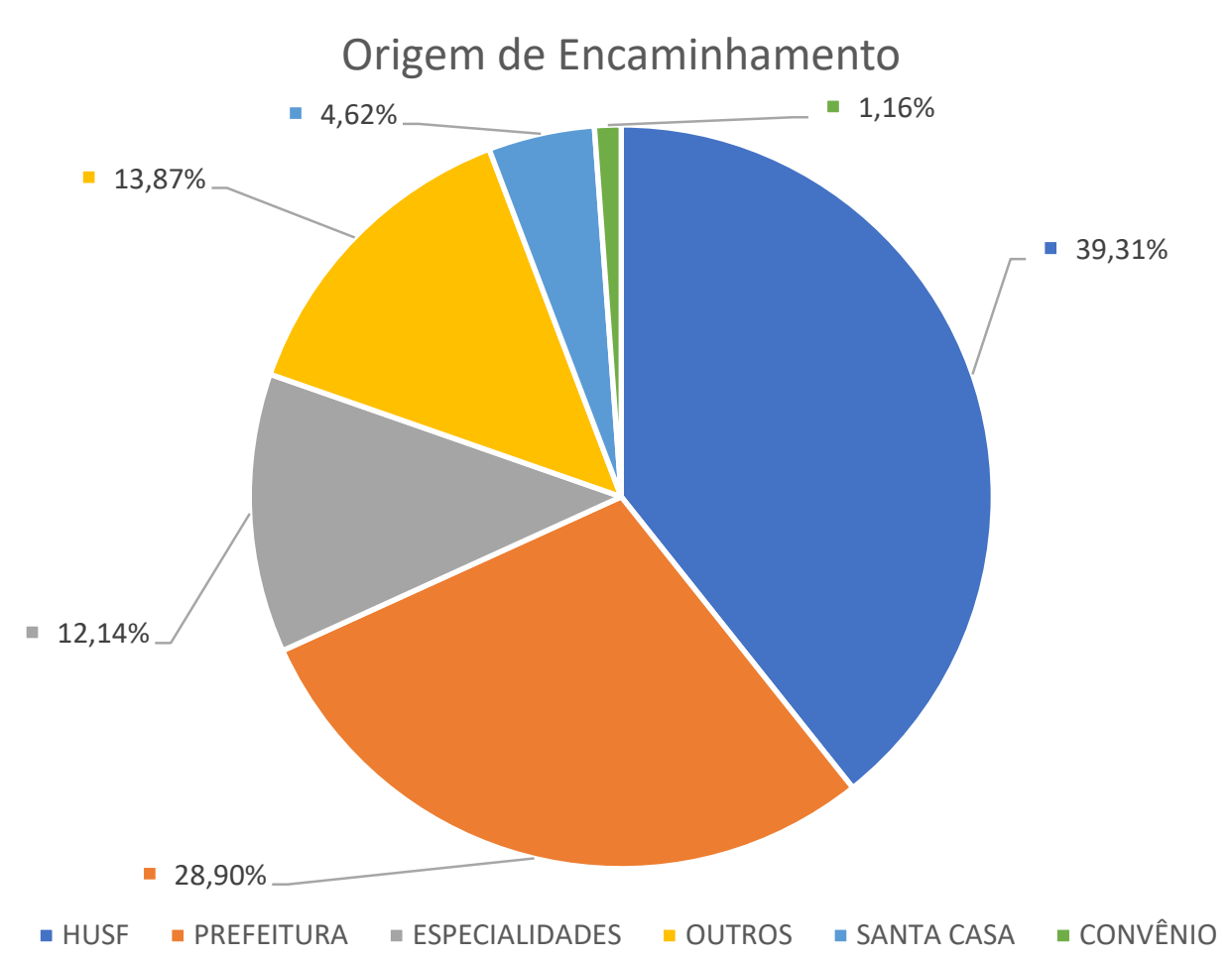

Figura 2 - Origem de Encaminhamentos.

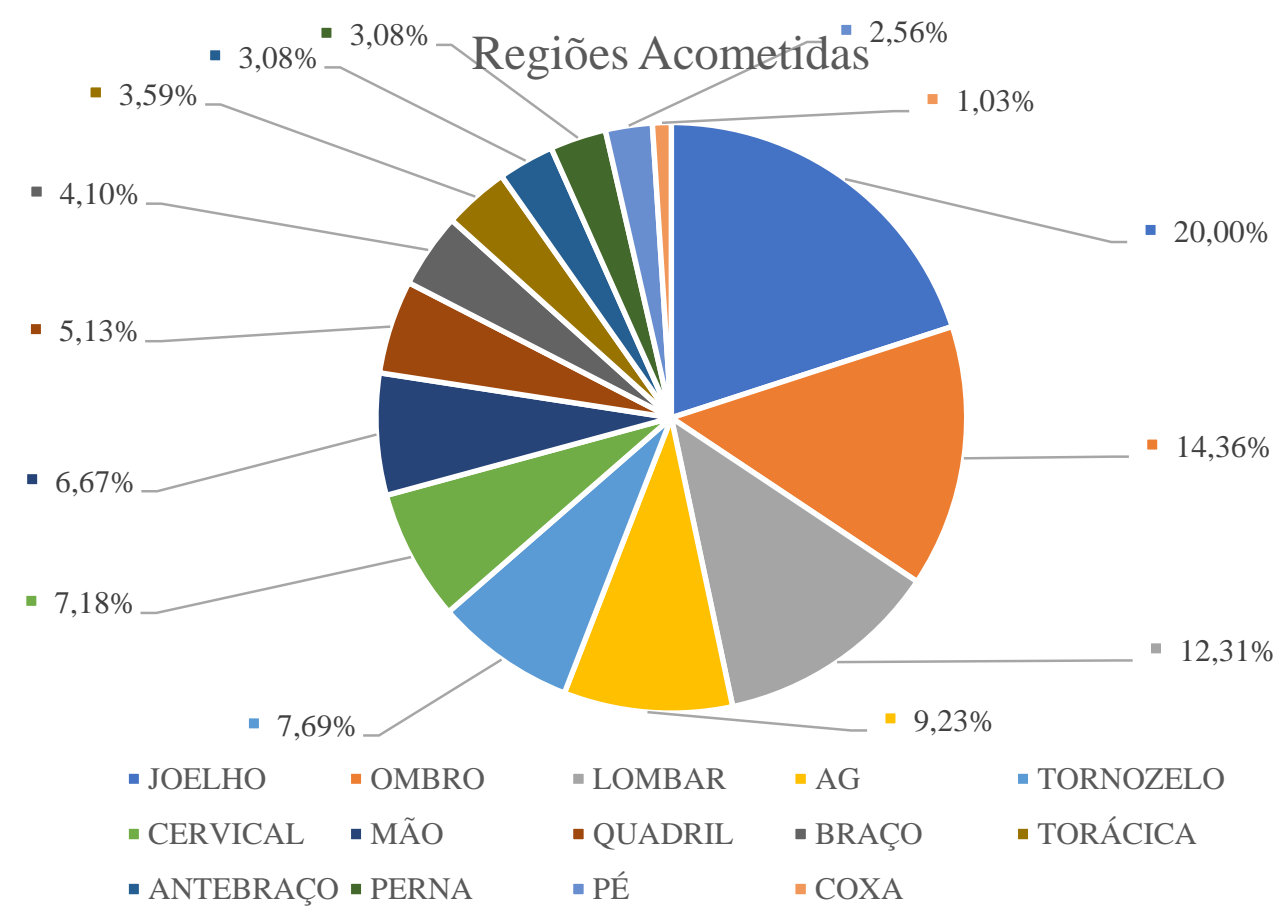

Figura 3-Regiões Acometidas. 
Destaca-se também as patologias (diagnósticos) mais incidentes em cada região corpórea, como observado na Tabela 1 , sendo a cervicalgia mais incidente na região cervical, a tendinopatia na região do ombro, as fraturas de úmero na região de braço, temos a fratura de antebraço (rádio/ulna) como a única incidente na região de antebraço, as fraturas foram também as mais incidentes na região da mão, a lombalgia foi a patologia mais incidente na região lombar, na coluna torácica temos a escoliose como mais incidente, a artroplastia total de quadril é a patologia mais incidente na região do quadril, na região de coxa temos como incidências iguais as osteossintes pós-fratura e as fraturas, na região do joelho, a região mais acometida temos a gonartrose como mais incidentes. Na região na perna as fraturas de tíbia e fíbula são as mais presentes nos pacientes, a região do tornozelo apresenta a entorse como caso mais incidente. No pé temos as fraturas como a patologia mais incidente nos casos analisados. Já nos acometimentos generalizados temos a osteoartrose como patologia mais incidente. Se somadas, as fraturas aparecem como a patologia mais incidente entre os prontuários analisados, somando 31 casos.

Tabela 1 - Quadro de Patologias por Região de Acometimento

\begin{tabular}{|c|c|}
\hline REGIÃO/PATOLOGIA & INCIDÊNCIA (\%) \\
\hline \multicolumn{2}{|l|}{ REGIÃO CERVICAL } \\
\hline - Cervicalgia & $6(42,86 \%)$ \\
\hline - Cervicobraquialgia & $5(35,71 \%)$ \\
\hline - Hérnia Discal & $1(7,14 \%)$ \\
\hline - Osteoartrose & $1(7,14 \%)$ \\
\hline - Escoliose & $1(7,14 \%)$ \\
\hline \multicolumn{2}{|l|}{ REGIÃO DO OMBRO } \\
\hline - Tendinopatia & $10(35,71 \%)$ \\
\hline - Lesão Manguito Rotador & $7(25,00 \%)$ \\
\hline - Bursite & $4(14,29 \%)$ \\
\hline - Síndrome do Impacto & $3(10,71 \%)$ \\
\hline - Luxação Acromioclavicular & $1(3,75 \%)$ \\
\hline - Capsulite Adesiva & $1(3,75 \%)$ \\
\hline - P.O Lesão de Supraespinhal & $1(3,75 \%)$ \\
\hline - Tenorrafia & $1(3,75 \%)$ \\
\hline \multicolumn{2}{|l|}{ REGIÃO DO BRAÇO } \\
\hline - Fraturas & $7(87,50 \%)$ \\
\hline - Subluxação & $1(12,50 \%)$ \\
\hline \multicolumn{2}{|l|}{ REGIÃO DO ANTEBRAÇO } \\
\hline - Fraturas & $6(100 \%)$ \\
\hline \multicolumn{2}{|l|}{ REGIÃO DA MÃO } \\
\hline - Fraturas & $6(46,15 \%)$ \\
\hline - Síndrome do Túnel do Carpo & $4(30,77 \%)$ \\
\hline - Tenossinovites & $3(23,08 \%)$ \\
\hline \multicolumn{2}{|l|}{ REGIÃO TORÁCICA } \\
\hline - Escoliose & $4(57,14 \%)$ \\
\hline - Síndrome do Desfiladeiro Torácico & $1(14,29 \%)$ \\
\hline - Discinesia Escapular & $1(14,29 \%)$ \\
\hline - Espondiloartrose & $1(14,29 \%)$ \\
\hline \multicolumn{2}{|l|}{ REGIÃO LOMBAR } \\
\hline - Lombalgia & $10(41,67 \%)$ \\
\hline - Lombociatalgia & $6(25,00 \%)$ \\
\hline - Hérnia Discal & $5(20,83 \%)$ \\
\hline - Osteoartrose Lombossacral & $2(8,33 \%)$ \\
\hline - Escoliose & $1(4,17 \%)$ \\
\hline
\end{tabular}

Cont.

Cont. Tabela 1 


\begin{tabular}{|c|c|}
\hline REGIÃO/PATOLOGIA & INCIDÊNCIA (\%) \\
\hline \multicolumn{2}{|l|}{ REGIÃO DO QUADRIL } \\
\hline - Artroplastia Total de Quadril & $5(50,00 \%)$ \\
\hline - Bursite Trocantérica & $2(20,00 \%)$ \\
\hline - Pubalgia & $1(10,00 \%)$ \\
\hline - Síndrome do Piriforme & $1(10,00 \%)$ \\
\hline - Displasia de Quadril & $1(10,00 \%)$ \\
\hline \multicolumn{2}{|l|}{ REGIÃO DA COXA } \\
\hline - Fraturas & $1(50,00 \%)$ \\
\hline - Osteossínteses Pós Fratura & $1(50,00 \%)$ \\
\hline \multicolumn{2}{|l|}{ REGIÃO DO JOELHO } \\
\hline - Gonartrose & $13(33,33 \%)$ \\
\hline - Condromalácia & $6(15,38 \%)$ \\
\hline - Artroplastia Total de Joelho & $6(15,38 \%)$ \\
\hline - Ligamento Cruzado Anterior & $3(7,69 \%)$ \\
\hline - Condropatia & $3(7,69 \%)$ \\
\hline - Artroscopia & $3(7,69 \%)$ \\
\hline - Tendinopatia Patelar & $1(2,56 \%)$ \\
\hline - Artropatia Patelar & $1(2,56 \%)$ \\
\hline - Disfunção Femoropatelar & $1(2,56 \%)$ \\
\hline - Entorse & $1(2,56 \%)$ \\
\hline - Lesão Meniscal & $1(2,56 \%)$ \\
\hline \multicolumn{2}{|l|}{ REGIÃO DA PERNA } \\
\hline - Fraturas & $4(66,67 \%)$ \\
\hline - Osteossíntese Pós Fratura & $1(16,67 \%)$ \\
\hline - Tendinopatia & $1(16,67 \%)$ \\
\hline \multicolumn{2}{|l|}{ REGIÃO DE TORNOZELO } \\
\hline - Entorse & $6(40,00 \%)$ \\
\hline - Fratura & $5(33,33 \%)$ \\
\hline - Lesão Ligamentar & $3(20,00 \%)$ \\
\hline - Tendinose & $1(6,67 \%)$ \\
\hline \multicolumn{2}{|l|}{ REGIÃO DO PÉ } \\
\hline - Fraturas & $2(40,00 \%)$ \\
\hline - Fasceíte Plantar & $1(20,00 \%)$ \\
\hline - Pós-Operatório de Hálux Valgo & $1(20,00 \%)$ \\
\hline - Lesão Ligamentar & $1(20,00 \%)$ \\
\hline \multicolumn{2}{|l|}{ ACOMETIMENTOS GENERALIZADOS } \\
\hline - Osteoartrose & $7(38,89 \%)$ \\
\hline - Artrite Reumatóide Juvenil & $3(16,67 \%)$ \\
\hline - Politrauma & $2(11,11 \%)$ \\
\hline - Fibromialgia & $1(5,56 \%)$ \\
\hline - Osteoporose & $1(5,56 \%)$ \\
\hline - Discopatia & $1(5,56 \%)$ \\
\hline - Hemiparesia & $1(5,56 \%)$ \\
\hline - Ressecção Sinovial & $1(5,56 \%)$ \\
\hline - Síndrome do Complexo Regional & $1(5,56 \%)$ \\
\hline
\end{tabular}

Observa-se na Figura 4 que o tipo de tratamento mais usado na reabilitação dos pacientes atendidos na Clínica-escola de Fisioterapia da Universidade São Francisco, excetuando-se a avaliação que é feita em absolutamente todos os pacientes, temos a modalidade cinesioterapia $(n=88)$ que é abordada através de recursos mecânicos e manuais de fortalecimento, alongamentos e técnicas utilizadas no processo de reabilitação. Logo em seguida temos os recursos da eletroterapia $(n=51)$, aparelhos usados para realizar analgesia, diminuir edemas, controlar processos inflamatórios, apoiar e favorecer a cicatrização de tecidos lesados ou realizar treinamentos neuromusculares. Outra modalidade de tratamento, as terapias manuais $(\mathrm{n}=34)$ que incluem aplicação dos métodos de Klapp, Maitland, Mulligan, Técnicas Quiropráticas e Osteopáticas, e as Mobilizações Neurais, entre outras. Os recursos da 
termofototerapia $(n=29)$ são os recursos utilizados para controle de espasmos, contraturas, melhoria na condição de elasticidade dos tecidos viscoelásticos, analgesia e aceleração no processo de cicatrização. A massoterapia $(n=17)$ consiste em técnicas de massagem realizadas para relaxamento muscular, liberações miofasciais e alongamentos utilizados nos pacientes atendidos na clínica. Ainda, o recurso de hidroterapia $(n=5)$ onde os pacientes são tratados no turbilhão, utilizando dos recursos terapêuticos que a água quente traz para benefício do paciente, e o Pilates $(\mathrm{n}=2)$. A média de sessões verificada foi de 11 sessões desde o dia de avaliação até a alta fisioterapêutica, variando de 2 sessões até 78 sessões, até o fechamento da coleta de dados deste estudo.

Os resultados referentes aos locais de incidência das patologias são coincidentes com um trabalho realizado por MARGOTTI (2003), onde constatou que dos 555 pacientes atendidos Universidade do Sul de Santa Catarina, os problemas de ombro, seguidos de lombalgias tiveram maior incidência de acometimentos, portanto encaminhados para tratamento. $\mathrm{O}$ autor ainda cita que problemas da coluna lombar estavam em segundo lugar nos casos levantados, sendo no presente estudo os problemas na coluna lombar ocupando a $3^{\text {a }}$ posição, enquanto SILVA; LIMA; LEROY (2013) descrevem em sua análise de 59 prontuários uma maior prevalência de patologias na região da lombar, sendo a lombalgia/lombociatalgia a patologia mais incidente, comprovando que não temos uma patologia mais incidente nas clinicas de fisioterapia, variando de local para local.

O estudo de GHISLENII et al. (2014) descreve uma maior prevalência de reabilitação pós fraturas, o que vai de acordo com o presente estudo, onde somadas, as fraturas de membros foram a de maior incidência.

A fila de espera do setor de Ortopedia/Traumatologia e Reumatologia conta com diversos tipos de patologia, citados já em nossos gráficos acima, totalizando 19,75\% do número total de prontuários analisados.

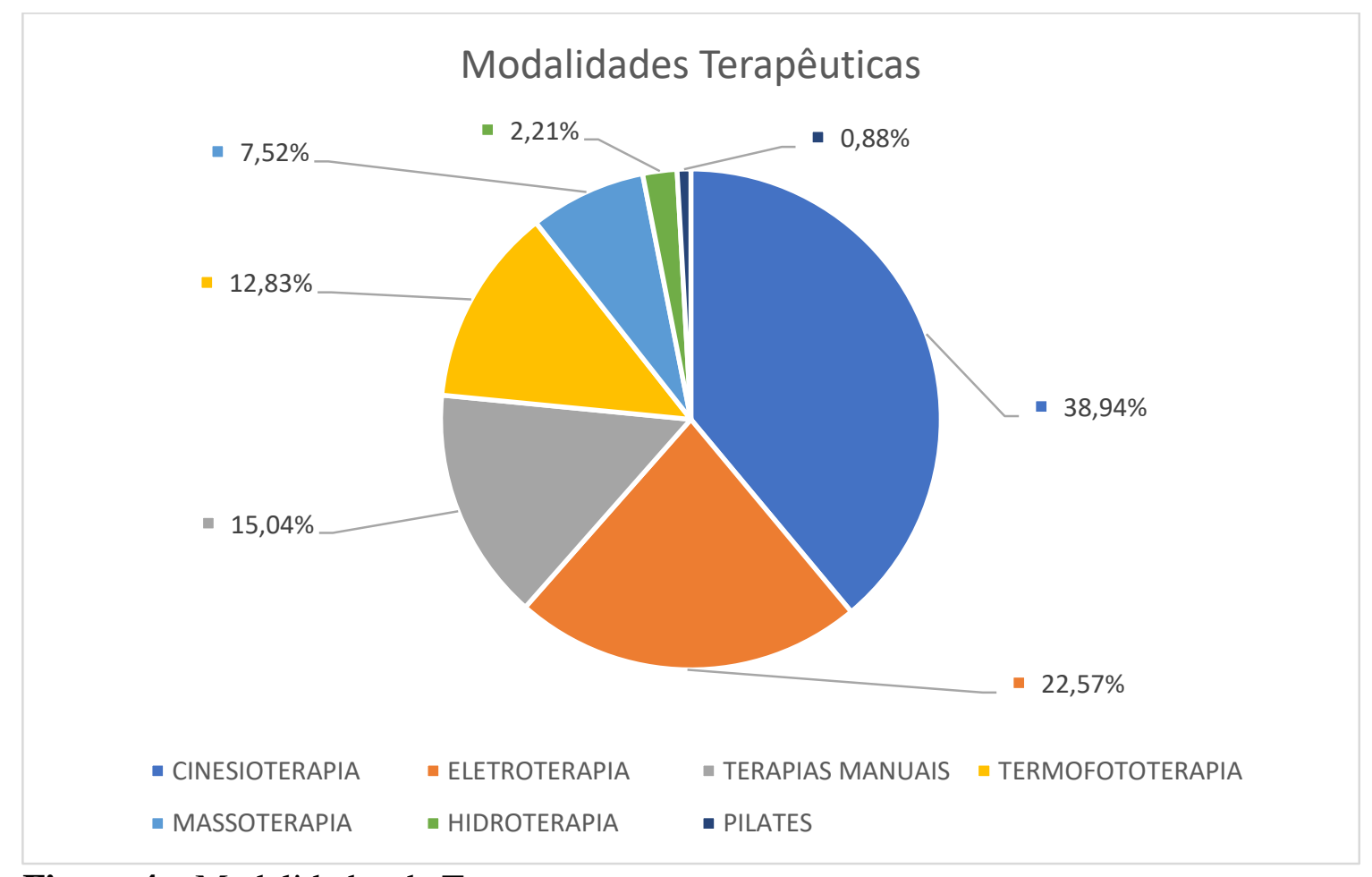

Figura 4 - Modalidades de Tratamento.

\section{CONCLUSÃO}


Conclui-se que o perfil do paciente atendido na Clínica-Escola de Fisioterapia da Universidade São Francisco é do gênero feminino com acometimento do membro inferior, sendo prioritariamente encaminhado pelo serviço do HUSF.

\section{REFERÊNCIAS}

BICALHO, G. G.; BARROS FILHO, A. A. Iniciação Científica: Como Elaborar em Projeto de Pesquisa. Rev. Ciências Médicas, Campinas, v.12, n.4, p.365-373, 2003.

BARBOSA RI, et. al. Perfil dos pacientes com lesões traumáticas do membro superior atendidos pela fisioterapia em hospital do nível terciário. Acta Fisiátr. v.20, n.1, p.14-19, 2013.

CARDOSO LGRA. Estudo sobre a distribuição dos serviços de reabilitação: o caso do estado do Rio de Janeiro. [dissertação]. Brasília: Escola Nacional de Saúde Pública; 2004.

CHAMLIAN, TS et al. Perfil Epidemiológico dos pacientes amputados de membros inferiores atendidos no Lar Escola São Francisco entre 2006 e 2012, Acta Fisiatr, v.20, n.4, p.219-223, 2013.

COELHO, R.R. Cap. 22 Doenças degenerativas e reumáticas do sistema locomotor em idosos. In: COELHO, R.R.Fisioterapia geriátrica, ed. 1, 2005.

CONELLY, L. B.; WOOLF, A.; BROOKS, P. Cost-effectiveness of interventions for musculoeskeletal conditions. In: JAMINSON, D. T. et al. Disease Control Priorities in Developing Countries. 2. ed., 2006

FUNCK, K.T; ESTIVALET, P.S. O perfil epidemiológico dos pacientes atendidos pelo serviço público de fisioterapia no município de Boa Vista do Cadeado, RS. Fisioter. Mov., v.28, n.4, p.685-692, Oct/Dec, 2015.

GHISLENI M.M, SILVA V.C.C, SANTOS M.V. Perfil epidemiológico dos pacientes atendidos na área de ortopedia e traumatologia da clínica-escola de fisioterapia Univates. Revista Destaques Acadêmicos, vol. 6, n.3, p.117-125, 2014.

GRAFFITHS, I. D. Introduction, In GRAFFITHS, Musculoskeletal disorders Medicine, 2002.

GOTLIEB SLD; LEURENTI R; JORGE MHPM. Mensuração em saúde pública. Saúde pública - bases conceituais. São Paulo: Ateneu, p.35-43. 2008.

Institucional. Serviços à comunidade - Clínica de Fisioterapia, disponível em: https://www.usf.edu.br/institucional/86367626,8/clinica+de+fisioterapia+.htm - acessado em 08/06/2017.

LEMOS C.A.G et al. Perfis das vítimas e tratamento de lesões por causas externas segundo atendimento pelo Centro de Reabilitação Municipal de Uberlândia, MG - Causas externas e fisioterapia, Revista Brasileira de Epidemiologia, Uberlândia, v.16, n.2, p.482-92, 2013. 
MARGOTTI, W., Prevalência dos dez distúrbios ortopédicos mais frequentes na clínica Escola de Fisioterapia da UNISUL, Trabalho de conclusão de curso, Universidade do Sul de Santa Catarina, 2003

OLIVEIRA, A.C; BRAGA, D.L.C. Perfil Epidemiológico dos pacientes na clínica de ortopedia da Universidade Paulista, J Health Sci Inst., Jundiaí, v.28, n.4, p.356-358, 2010.

RAMOS et al. Perfil das crianças e adolescentes atendidos na clínica escola de fisioterapia da UESB. Revista Baiana de Saúde Pública, v.36, n.2, p.386-395 abr/jun, 2012.

RIBEIRO CTM, Ribeiro MG, Araújo AP, Mello LR, Rubim LC, Ferreira JES. O sistema público de saúde e as ações de reabilitação no Brasil. Rev Panam Salud Pública, v.28, n.1, 43-8, 2010.

SANTOS et al. Perfil sócio demográfico e físico-funcional de pacientes com lombalgia atendidos em Manaus/AM, Rev. Dor São Paulo, v.16, n.4, p272-275, out-dez, 2015.

SILVA, G.G; SIRENA, S.A. Perfil dos encaminhamentos a fisioterapia por um serviço de Atenção Prímaria à Saúde, Epidemiol. Serv. Saúde, Brasília, Porto Alegre, v.24, n.1, p.123133, jan-mar 2012.

SILVA; LIMA; LEROY. Perfil epidemiológico dos pacientes assistidos na clínica de fisioterapia traumato-ortopédica da prefeitura de Hidrolândia, Revista Movimenta, v.6, n.3, 2013.

SILVA, K.O.C et. al. Perfil dos pacientes atendidos na clínica escola de fisioterapia no setor de ortopedia e traumatologia, Revista Eletrônica Estácio Saúde, Rio Grande do Norte, vol.4, n.1, p.53-58,2015. Disponível em http://revistaadmmade.estacio.br/index.php/saudesantacatarina/article/viewFile/1481/698.

Acesso em 26 set. 2016

UENO HM, Natal D. Fundamentos da epidemiologia. In: Rocha AA, Cesar CLG. Saúde Pública - bases conceituais. São Paulo: Ateneu, n.2, p.15-34, 2008.

UNIVERSIDADE SÃO FRACISCO. Projeto pedagógico do curso de Fisioterapia da Universidade São Francisco. Bragança Paulista, vol I, 2013

WATANABE, L.A. Perfil epidemiológico dos pacientes atendidos no setor de fisioterapia de uma clínica de ortopedia em Goiânia. Trabalho de Especialização em Fisioterapia Musculoesquelética, Universidade São Marcos Goiânia, 2012. 